\title{
SIGN CHANGES IN SUMS OF THE LIOUVILLE FUNCTION
}

\author{
PETER BORWEIN, RON FERGUSON, AND MICHAEL J. MOSSINGHOFF
}

\begin{abstract}
The Liouville function $\lambda(n)$ is the completely multiplicative function whose value is -1 at each prime. We develop some algorithms for computing the sum $T(n)=\sum_{k=1}^{n} \lambda(k) / k$, and use these methods to determine the smallest positive integer $n$ where $T(n)<0$. This answers a question originating in some work of Turán, who linked the behavior of $T(n)$ to questions about the Riemann zeta function. We also study the problem of evaluating Pólya's sum $L(n)=\sum_{k=1}^{n} \lambda(k)$, and we determine some new local extrema for this function, including some new positive values.
\end{abstract}

\section{INTRODUCTION}

The Liouville function $\lambda(n)$ is the completely multiplicative function defined by $\lambda(p)=-1$ for each prime $p$. Let $\zeta(s)$ denote the Riemann zeta function, defined for complex $s$ with $\Re(s)>1$ by

$$
\zeta(s)=\sum_{n \geq 1} \frac{1}{n^{s}}=\prod_{p}\left(1-\frac{1}{p^{s}}\right)^{-1} .
$$

It follows then that

$$
\frac{\zeta(2 s)}{\zeta(s)}=\prod_{p}\left(1+\frac{1}{p^{s}}\right)^{-1}=\sum_{n \geq 1} \frac{\lambda(n)}{n^{s}}
$$

for $\Re(s)>1$.

Let $L(n)$ denote the sum of the values of the Liouville function up to $n$,

$$
L(n):=\sum_{k=1}^{n} \lambda(k)
$$

so that $L(n)$ records the difference between the number of positive integers up to $n$ with an even number of prime factors and those with an odd number (counting multiplicity). Pólya noted in 1919 15 that the Riemann hypothesis follows if $L(n)$ does not change sign for sufficiently large $n$. This may be established by applying partial summation in (11) and then employing a well-known theorem of Landau on the convergence of Dirichlet series with terms of constant sign; see for instance 14. It is also known that the zeros of the zeta function are all simple if $L(n)$ is eventually of constant sign.

Received by the editor July 7, 2006 .

2000 Mathematics Subject Classification. Primary 11Y35; Secondary 11M26.

Key words and phrases. Liouville function, Pólya's sum, Turán's sum, Riemann hypothesis.

The research of P. Borwein was supported in part by NSERC of Canada and MITACS. 
Pólya proved in [15] that $L((p-3) / 4)=0$ for any prime $p>7$ for which $p \equiv 3$ mod 4 and the number of classes of positive definite quadratic forms of discriminant $-p$ is 1 , so $L((p-3) / 4)=0$ for $p=11,19,43,67$, and 163 . He verified that $L(n) \leq 0$ for all $n$ between 2 and approximately 1500, and found that in fact $L(n)=0$ for several values of $n$ beyond those obtained from the quadratic imaginary fields with class number 1 ; for example, $L(586)=0$.

In 1940, Gupta [6] verified that $L(n) \leq 0$ for $2 \leq n \leq 20000$. Also, D. H. Lehmer's review of Gupta's paper mentions that $L(48512)=-2$, so it seems possible that Lehmer may have performed some extensive computations on this problem around this time, too. Haselgrove [7] later verified that $L(n)$ remains negative up to 250000 , and in his article [8] he remarks that Lehmer had in fact checked this condition up to $n=600000$.

In 1942, Ingham [10] noted that the Riemann hypothesis, and the simplicity of the zeros of $\zeta(s)$ follow more generally if either $L(n)<c \sqrt{n}$ or $L(n)>-c \sqrt{n}$, for some positive constant $c$. Further, it would also follow from either of these conditions that the imaginary parts of the zeros of $\zeta(s)$ lying in the upper half-plane would satisfy infinitely many nontrivial linear relations over the field of rationals.

Similar statements hold for a particular weighted sum of the Liouville function. Landau proved in his thesis that the convergence of the sum

$$
\sum_{n \geq 1} \frac{\lambda(n)}{n}=0
$$

is in fact equivalent to the prime number theorem. (Landau also considered the analogous statement involving the Möbius function, $\mu(n)$.) Define the function $T(n)$ by

$$
T(n):=\sum_{k=1}^{n} \frac{\lambda(k)}{k},
$$

and let $U_{n}(s)$ denote the sum of the first $n$ terms of the series for the zeta function,

$$
U_{n}(s):=\sum_{k=1}^{n} \frac{1}{k^{s}} .
$$

In 1948, Turán [18] explored connections between the values of $T(n)$ and $U_{n}(s)$, and their relation to the Riemann hypothesis. He proved that the Riemann hypothesis follows if $U_{n}(s)$ does not vanish in the half-plane $\Re(s)>1$ for sufficiently large $n$, or more generally in the half-plane

$$
\Re(s) \geq 1+\frac{c}{\sqrt{n}}
$$

for some positive constant $c$. Somewhat weaker conditions suffice as well (see [18] and [19). Since $\zeta(s)$ has no zeros in $\Re(s)>1$, these conditions may seem plausible. In fact, Turán proved that if $U_{n}(s)$ does not vanish in the half-plane (2), then

$$
T(n)>-\frac{c_{1}}{\sqrt{n}}
$$

for a positive constant $c_{1}$, and that this condition implies the Riemann hypothesis. In particular, therefore, the Riemann hypothesis follows if $T(n)$ remains positive for large $n$.

We remark that in 1983 Montgomery [13] established that $U_{n}(s)$ does in fact have zeros with large real part, proving that for each positive number $c<\frac{4}{\pi}-1$ 
and every $n>n_{0}(c)$, the function $U_{n}(s)$ has zeros in the half-plane $\Re(s)>1+$ $c(\log \log n) / \log n$.

In [18, Turán reported that the positivity of $T(n)$ was checked for $n \leq 1000$ by five Danish mathematicians: Eilertsen, Kristensen, Petersen, Poulsen, and Winther. In 1952, Larsen 11 corrected some minor errors in the previously reported values for $T(293)$ and $T(1000)$, and checked that $T(n)>0$ for $n \leq 4528$. The stopping point here was selected no doubt because $L(4528)=-74$, and $L(n)$ does not achieve a smaller value until $n=6317$. We mention however that Larsen's reported value for $T(4528)$ is not correct (its actual value is $.0035514 \ldots$ ), and that his reported minimum over this range at $n=2837$ is erroneous as well. In fact, Larsen remarked that his value for $T(2837)$ is something of a near miss, since he calculated it to be less than $1 / 2837$. However, only $n=3,8,13$, and 32 have the property that $T(n)<1 / n$, at least until shortly before the first sign change of $T(n)$, described later in this article.

In 1953, Bateman and Chowla 22 remarked that the condition $T(n)>0$ was checked up to $n=100000$ at the Institute for Numerical Analysis, a laboratory affiliated with the National Bureau of Standards that existed at UCLA between 1947 and 1954. It is not mentioned who performed these calculations, but it seems likely that it was again D. H. Lehmer, who was involved with computations at the Institute around this time. (Lehmer's review of the article of Bateman and Chowla in fact includes some additional information on the calculation to 100000 , namely, that the computations were performed on the National Bureau of Standards Western Automatic Computer, known as the SWAC [9. 1)

In 1958, Haselgrove proved that both $L(n)$ and $T(n)$ change sign 8 . We briefly describe his proof, which builds on the work of Ingham [10. Assume that the Riemann hypothesis is valid, and that the zeros of the zeta function are all simple. Let $\left\{\rho_{n}\right\}$ denote the sequence of zeros of $\zeta(s)$ on the critical line in the upper halfplane, and write $\rho_{n}=\frac{1}{2}+i \gamma_{n}$, with $\gamma_{n}<\gamma_{n+1}$ for $n \geq 1$. Define the function $A(x)$ by

$$
A(x):=e^{-x / 2} L\left(e^{x}\right),
$$

and for a positive real number $m$, define the function $A_{m}^{*}(x)$ by

$$
A_{m}^{*}(x):=\frac{1}{\zeta(1 / 2)}+2 \Re\left(\sum_{0<\gamma_{n}<m}\left(1-\frac{\gamma_{n}}{m}\right) \frac{\zeta\left(2 \rho_{n}\right)}{\rho_{n} \zeta^{\prime}\left(\rho_{n}\right)} e^{i \gamma_{n} x}\right) .
$$

Then, for any fixed positive real numbers $m$ and $y$, Ingham proved that

$$
\liminf _{x \rightarrow \infty} A(x) \leq \liminf _{x \rightarrow \infty} A_{m}^{*}(x) \leq A_{m}^{*}(y) \leq \limsup _{x \rightarrow \infty} A_{m}^{*}(x) \leq \limsup _{x \rightarrow \infty} A(x) .
$$

Therefore, if one can find values for $m$ and $y$ for which $A_{m}^{*}(y)>0$, then it follows that $L(n)>0$ for infinitely many integers $n$. (Naturally, one obtains the same conclusion if the Riemann hypothesis is false, or if its zeros are not all simple.) Haselgrove found that selecting $m=1000$ and $y=831.847$ produces $A_{m}^{*}(y) \approx$ .00495. This computation employed the first 649 zeros of the Riemann zeta function.

Turán's problem was investigated in the same way. Define

$$
B(x):=e^{x / 2} T\left(e^{x}\right)
$$

\footnotetext{
${ }^{1}$ The SWAC was employed in a number of computations in number theory around this time, including Robinson's determination of the first five Mersenne primes found with the aid of a computer [16].
} 
and let

$$
B_{m}^{*}(x):=-\frac{1}{\zeta(1 / 2)}+2 \Re\left(\sum_{0<\gamma_{n}<m}\left(1-\frac{\gamma_{n}}{m}\right) \frac{\zeta\left(2 \rho_{n}\right)}{\left(\rho_{n}-1\right) \zeta^{\prime}\left(\rho_{n}\right)} e^{i \gamma_{n} x}\right) .
$$

Then inequalities analogous to those in (5) hold for the functions $B(x)$ and $B_{m}^{*}(x)$, and Haselgrove found that selecting $m=1000$ and $y=853.853$ or $y=996.980$ produces a negative value of $B_{m}^{*}(y)$. It follows that $T(n)<0$ for infinitely many integers $n$. Haselgrove's results, however, do not identify a specific value of $n$ where $L(n)>0$ or $T(n)<0$.

Lehman investigated Pólya's problem in 1960 [12. He proved that the function $A^{*}(x)$ represents a smoothing of the function $A(x)$, provided the Riemann hypothesis holds and that there exists a constant $c$ in $(0,1)$ such that $1 / \zeta^{\prime}(\rho)=O\left(|\rho|^{c}\right)$ whenever $\rho$ is a complex zero of $\zeta(s)$. For example, Figure 1(a) shows a plot of $A(x)$ for several hundred sampled values of $L\left(e^{x}\right)$ over $22 \leq x \leq 23$, and Figure 1(b) exhibits $A_{10000}^{*}(x)$ over the same interval. Figures 2(a) and 2(b) show that $B_{m}^{*}(x)$ approximates $B(x)$ in the same way.

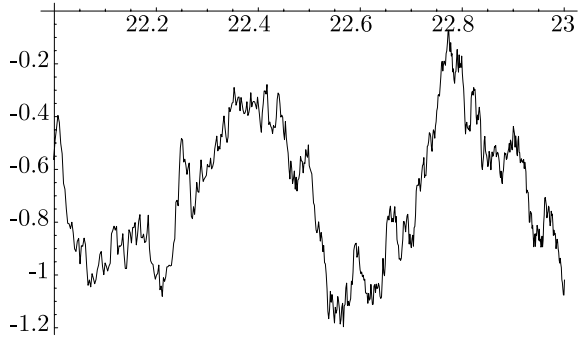

(a) $A(x)$

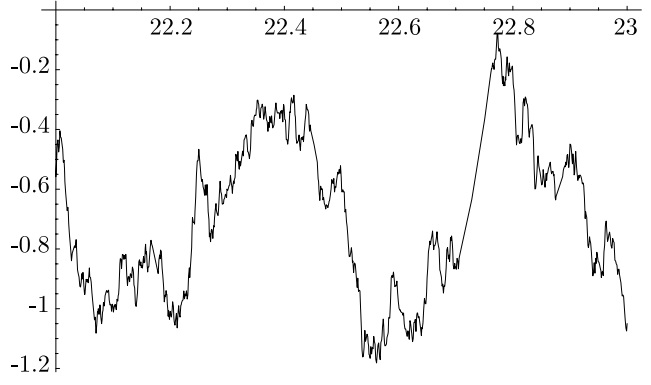

(b) $A_{10000}^{*}(x)$

Figure 1. $A(x)$ and $A_{10000}^{*}(x)$ for $22 \leq x \leq 23$

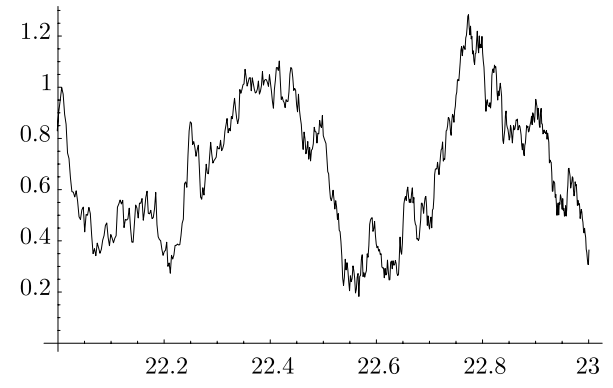

(a) $B(x)$

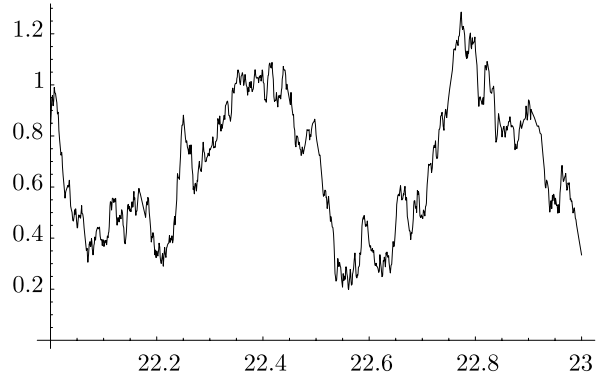

(b) $B_{10000}^{*}(x)$

FIGURE 2. $B(x)$ and $B_{10000}^{*}(x)$ for $22 \leq x \leq 23$

By investigating the functions $A_{m}^{*}(x)$ for $m \leq 1000$, Lehman suspected that $L(n)$ changes sign near $n=9.05 \cdot 10^{8}$. He developed an algorithm for computing $L(n)$ for a particular value of $n$ quickly, and used this method to determine that 
in fact $L(906180359)=1$. This algorithm is described in section 3 Lehman also recorded that $L(906400000)=708$, and Anderson and Stark [1] used this datum to show that there exist infinitely many integers $n$ for which

$$
L(n)>.023519 \sqrt{n} .
$$

In 1980, Tanaka 17] determined that $n=906150257$ is the smallest integer $n>1$ for which $L(n)=1$. Tanaka also reported that $L(n) \leq 829$ for $n \leq 10^{9}$, although he did not report that this value occurred at $n=906316571$. With this information, the method of Anderson and Stark shows that

$$
L(n)>.027536 \sqrt{n}
$$

infinitely often. The value of the constant here is simply $829 / \sqrt{906316571}$.

Precise information on negative values of $T(n)$, however, have remained unknown. In this paper, we determine the smallest positive integer $n$ for which $T(n)<0$. We also determine the minimum value of $T(n)$ for $n \leq 7.5 \cdot 10^{13}$. We establish the following theorem in section 2

Theorem 1. Let $T(n)$ denote the sum $\sum_{k=1}^{n} \lambda(k) / k$. The smallest positive integer $n$ for which $T(n)<0$ is $n=72185376951205$, and the minimal value of $T(n)$ for $n \leq 7.5 \cdot 10^{13}$ is $T(72204113780255) \approx-2.0757641 \cdot 10^{-9}$.

This minimal value was recently used by Granville and Soundararajan [5] to determine an explicit bound on negative values of the truncation of the series for $L(1, \chi)$, where $L(s, \chi)$ is the $L$-function associated with the quadratic Dirichlet character $\chi$. The relationship between Turán's problem and truncations of $L(1, \chi)$ for real Dirichlet characters is also discussed by Bateman and Chowla [2] and Wiener and Wintner 20.

In section 3 we describe our implementation of Lehman's method for computing $L(n)$. We use this algorithm to determine some additional values where $L(n)>0$. These results allow us to strengthen inequality (9) immediately. We establish the following theorem.

Theorem 2. There exist infinitely many positive integers $n$ for which

$$
L(n)>.061867 \sqrt{n} .
$$

\section{Sign Changes in TurÁN'S SUM}

Figure 3 exhibits the function $B_{25000}^{*}(x)$ near $x=31.91$, and indicates that $T(n)$ may change sign near $n=\exp (31.9105) \approx 7.22 \cdot 10^{13}$. We develop some algorithms to establish that the first sign change for $T(n)$ indeed occurs near this value.

2.1. Constructing a table of values for $\lambda(n)$. To facilitate our computation of $L(n)$ and $T(n)$, we first construct a table of values of the Liouville function up to a given integer, $N$. Clearly, only one bit is needed to indicate the value of $\lambda(k)$ for each integer $k$. We find it convenient to use the 0 bit to indicate $\lambda(k)=1$ and the 1 bit to indicate $\lambda(k)=-1$. It is an inefficient use of memory, however, to store the value of $\lambda(k)$ for each $k \leq N$, when one can exploit the simple relation $\lambda(k p)=-\lambda(k)$ for any prime $p$. By avoiding storage of the values of $\lambda(k)$ when $k$ is a multiple of 2,3 , or 5 , we reduce the size of the table to $4 N / 15$ bits. Of course, to compensate for this, a program using the table must remove all multiples of 2 , 3 , and 5 before looking up the value of the Liouville function on the remaining 


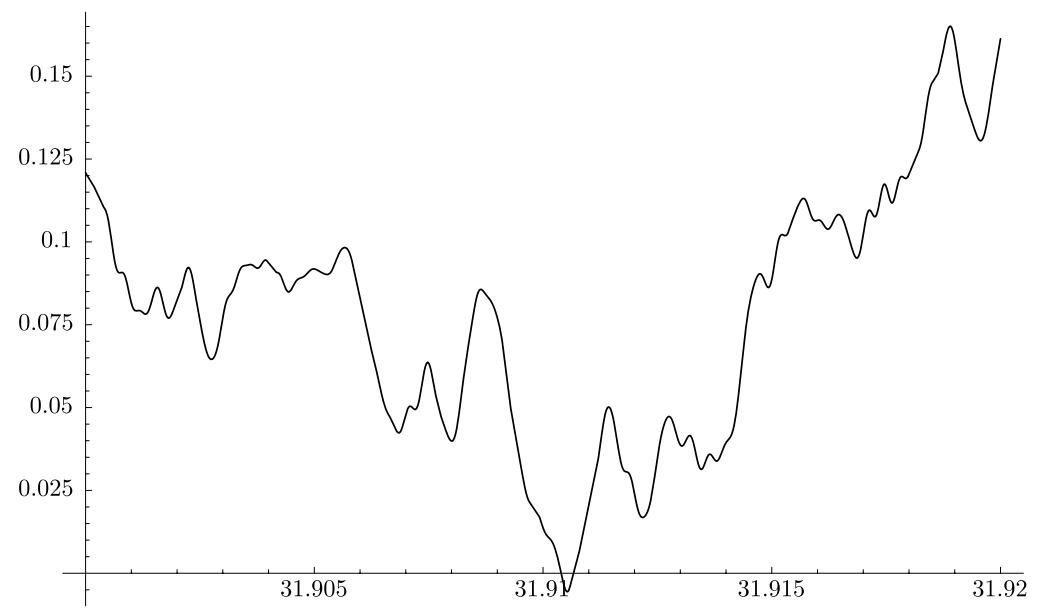

FIGURE $3 . B_{25000}^{*}(x)$ near $x=31.9$

part, but this imposes a reasonable balance of time and space requirements in our programs. Removing multiples of 2,3 , and 5 is particularly convenient, since then we only store eight bits for each block of 30 integers, and the smallest addressable block of memory on most computers is a byte consisting of eight bits.

Algorithm 1. Construction of a table of values for the Liouville function.

Input. A positive integer $N$, with $30 \mid N$.

Output. A table consisting of the values of $\lambda(n)$ for each integer $n$ in $[1, N]$ with Description. $\operatorname{gcd}(n, 30)=1$, one bit per value.

Step 1. Allocate $N / 30$ bytes of memory. Store 127 in the first byte and 255 in all subsequent bytes. Set $a:=30$.

Step 2. Set $b:=\min \{2 a-1, N-1\}$.

Step 3. For each prime $p$ with $7 \leq p \leq \sqrt{b}$, perform the following action: For each multiple $k$ of $p$ in $[a, b]$ with $\operatorname{gcd}(k, 30)=1$, set the bit representing $k$ in the table to $1-\beta$, where $\beta$ is the bit representing $\lambda(k / p)$.

Step 4. Set $a:=b+1$. If $a=N$ then save the table and quit; otherwise return to Step 2.

Note that the first byte in the table is 127 because $\lambda(1)=1$ and $\lambda(k)=-1$ for each $k$ with $1<k<30$ and $\operatorname{gcd}(k, 30)=1$, so the first eight bits in the table must be 01111111 . Also, we initialize every subsequent bit in the table with 1 because any bit left undisturbed by the algorithm necessarily represents a prime number.

2.2. Computing the values of $\lambda(n)$ across an interval. Next we describe an algorithm that employs a sieving strategy, aided by a table of values of $\lambda(k)$ produced by Algorithm 1 to compute sums involving the Liouville function over an interval. This algorithm is designed so that multiple machines can sieve different intervals independently. We define $L(a, b)$ to be the sum of the values of the Liouville 
function over the interval $[a, b]$,

$$
L(a, b):=\sum_{k=a}^{b} \lambda(k),
$$

and define $T^{+}(a, b)$ and $T^{-}(a, b)$ to be the sum of the positive and negative terms, respectively, in the Turán sum over $[a, b]$,

$$
\begin{aligned}
T^{+}(a, b) & :=\sum_{\substack{a \leq k \leq b \\
\lambda(k)=1}} \frac{1}{k}, \\
T^{-}(a, b) & :=\sum_{\substack{a \leq k \leq b \\
\lambda(\bar{k})=-1}} \frac{1}{k} .
\end{aligned}
$$

Algorithm 2 employs a sieving strategy to compute the values of the Liouville function over an interval. Unlike Algorithm 1, our table of values for $\lambda(n)$ requires two bits per integer, since three different states are possible for each integer in the block: $\lambda(n)$ is known and has value 1 (represented by the bit pattern 01), $\lambda(k)$ is known and has value -1 (bit pattern 10), and $\lambda(n)$ is unknown (bit pattern 00). Given positive integers $a_{0}, n$, and $M$, the algorithm computes the sums (10), (11), and (12) over the interval $\left[a_{0}, a_{0}+n M\right)$ by performing $n$ sieving computations, each on an interval of size $M$.

Algorithm 2. Computing the values of the Liouville function over an interval.

Input. Positive integers $a_{0}, M, N, n$, and $b$, with $4|M, 30| N$, and a table recording $\lambda(k)$ for all positive integers $k \leq N$ with $\operatorname{gcd}(k, 30)=1$.

Output. The values of $L\left(a_{0}, a_{0}+n M-1\right), T^{+}\left(a_{0}, a_{0}+n M-1\right), T^{-}\left(a_{0}, a_{0}+n M-1\right)$, the maximal and minimal values of $L\left(a_{0}, a_{0}+k\right)$ and $T^{+}\left(a_{0}, a_{0}+k\right)-$ $T^{-}\left(a_{0}, a_{0}+k\right)$ over $0 \leq k<n M$, and the values of $k$ where these extreme values occur. The values of $T^{+}$and $T^{-}$over the interval are computed to $b$ bits of precision.

Description.

Step 1. Read a table of values of the Liouville function up to $N$, created using Algorithm 1. Read a table of primes up to $\sqrt{a_{0}+n M}$. Allocate a table $I$ of size $2 M$ bits to record values of the Liouville function over an interval of size $M$.

Step 2. For each integer $m$ with $0 \leq m<n$, set $a:=a_{0}+m M$, set $b:=a+M-1$, set the bits in $I$ to indicate that $\lambda(k)$ is unknown for $a \leq k \leq b$, and perform Steps 3 through 6 . Then perform Step 7.

Step 3. For each prime $p$ satisfying $b /(N-1) \leq p \leq \sqrt{b}$, use a sieving strategy to test each multiple $k$ of $p$ in $[a, b]$. For each such $k$, if the value of $\lambda(k)$ is presently unknown, then since $k / p<N$, we may use the table to determine the value of $\lambda(k / p)$. This requires removing all factors of 2,3 , and 5 from $k$ and keeping track of the number of such factors. Set $\lambda(k)=-\lambda(k / p)$.

Step 4. For each prime $p$ from $\lceil b /(N-1)\rceil-1$ down to 7 , again use a sieving strategy to test each multiple $k$ of $p$ in $[a, b]$. If $\lambda(k)$ is presently unknown, then remove a factor of $p$ from $k$, as well as any factors of 2,3 , and 5 . 
Then use trial division to search for additional prime factors $q$ of $k$, beginning with $q=7$ and halting when either the remaining cofactor is less than $N$, or when all primes up to $p$ have been tested (in the latter case the remaining cofactor is prime). Use the count of prime divisors removed from $k$, together with the table of existing values for the Liouville function, to set the value for $\lambda(k)$.

Step 5. Scan the table for any remaining multiples of 2,3 , or 5 (these have the form $2^{r} 3^{s} 5^{t}$ or $2^{r} 3^{s} 5^{t} p$, with $p$ a large prime), and set $\lambda(k)$ for these numbers.

Step 6. Any remaining numbers $k$ in $[a, b]$ for which $\lambda(k)$ is unknown are in fact prime. Use the table $I$, together with the computations from the prior blocks, to determine $L\left(a_{0}, b\right), T^{+}\left(a_{0}, b\right), T^{-}\left(a_{0}, b\right)$, and the maximal and minimal values of $L\left(a_{0}, k\right)$ and $T^{+}\left(a_{0}, k\right)-T^{-}\left(a_{0}, k\right)$ for $a_{0} \leq k \leq b$.

Step 7. Save the values $L\left(a_{0}, a_{0}+n M-1\right), T^{+}\left(a_{0}, a_{0}+n M-1\right)$, and $T^{-}\left(a_{0}, a_{0}+\right.$ $n M-1)$ to a file, as well as the maximum and minimum values of $L\left(a_{0}, a_{0}+k\right)$ and $T^{+}\left(a_{0}, a_{0}+k\right)-T^{-}\left(a_{0}, a_{0}+k\right)$ over $0 \leq k<n M$, and the values of $k$ where these local extrema occur.

2.3. Results. We use Algorithm 2 to compute the sums $L(n)$ and $T(n)$ up to $7.5 \cdot 10^{13}$. This computation was performed using a cluster of PowerMac G5 workstations, each with $2.5 \mathrm{GHz}$ dual processors and two gigabytes of memory, at the center for Interdisciplinary Research in the Mathematical and Computational Sciences (IRMACS) at Simon Fraser University. We use a table of values of the Liouville function (for integers relatively prime to 30) produced by Algorithm 1 up to $N=4 \cdot 10^{10}+20$; this requires about 1.3 gigabytes of memory. Empirically, we find that $M=16000000$ is a good choice for the sieving block size (the somewhat small block size may allow more of the sieving interval to be held in cache). We use $b=192$ bits of precision in the computation of the Turán sums, using the GMP library 4 to perform these high-precision calculations. Each value $\lambda(n) / n$ is then computed to approximately 58 digits of precision, so our computation of $T(n)$ up to $7.5 \cdot 10^{13}$ is accurate to at least 44 decimal places.

The computation was divided into 1286 segments, each one running overnight on one processor. The first 380 partitions sieved $n=4000$ blocks of size $M$; the latter 905 segments used $n=3500$. The last process computed the values of the Pólya and Turán sums over the prefix $[1, N-1]$. These jobs were run over 30 nights on the IRMACS cluster, requiring about 2.5 years of CPU time in all.

We check the integrity of the data produced by these computations with several calculations. First, for each interval $[a, b]$ corresponding to a segment of the computation, we check if $T^{+}(a, b)+T^{-}(a, b)$ matches $\sum_{n=a}^{b} 1 / n$ to the required precision, computing the harmonic series independently to high precision by analytic means. Second, we use Lehman's algorithm, described in section 3, to check that the values of $L(a)$ and $L(b)$ are correct, as well as the maximum and minimum values attained by $L(n)$ over $[a, b]$.

We find that $T(n)$ first changes sign at

$$
n_{0}=72185376951205 \text {, }
$$


TABLE 1. Some local minima of $L(n)$ and $T(n)$.

\begin{tabular}{|ccc|ccc|}
\hline$n$ & $L(n)$ & $T(n)$ & $n$ & $L(n)$ & $T(n)$ \\
\hline 293 & -21 & $5.11228 \cdot 10^{-3}$ & 3847002655 & -68681 & \\
468 & -24 & & 4430947670 & -73436 & \\
684 & -28 & & 6321603934 & -96460 & \\
1132 & -42 & $2.81770 \cdot 10^{-3}$ & 10097286319 & -123643 & $1.38015 \cdot 10^{-6}$ \\
1760 & -48 & & 15511912966 & -158636 & $8.42129 \cdot 10^{-7}$ \\
2804 & -66 & $1.99019 \cdot 10^{-3}$ & 24395556935 & -172987 & \\
4528 & -74 & & 39769975545 & -238673 & $8.05229 \cdot 10^{-7}$ \\
7027 & -103 & $1.37995 \cdot 10^{-3}$ & 98220859787 & -365305 & $6.08024 \cdot 10^{-7}$ \\
9840 & -128 & $8.62126 \cdot 10^{-4}$ & 149093624694 & -461684 & $4.29564 \cdot 10^{-7}$ \\
24426 & -186 & & 217295584371 & -598109 & $2.20717 \cdot 10^{-7}$ \\
59577 & -307 & $4.97200 \cdot 10^{-4}$ & 341058604701 & -726209 & \\
96862 & -414 & $1.19960 \cdot 10^{-4}$ & 576863787872 & -900668 & \\
386434 & -698 & & 835018639060 & -1038386 & \\
614155 & -991 & $1.08828 \cdot 10^{-4}$ & 1342121202207 & -1369777 & $1.64664 \cdot 10^{-7}$ \\
925985 & -1253 & $5.46512 \cdot 10^{-5}$ & 2057920042277 & -1767635 & $1.00682 \cdot 10^{-7}$ \\
2110931 & -1803 & & 2147203463859 & -1784793 & $9.56193 \cdot 10^{-8}$ \\
3456120 & -2254 & & 3271541048420 & -2206930 & $8.51691 \cdot 10^{-8}$ \\
5306119 & -2931 & $4.10527 \cdot 10^{-5}$ & 4686763744950 & -2259182 & \\
5384780 & -2932 & & 5191024637118 & -2775466 & $6.31694 \cdot 10^{-8}$ \\
8803471 & -3461 & & 7934523825335 & -3003875 & \\
12897104 & -4878 & $3.05124 \cdot 10^{-6}$ & 8196557476890 & -3458310 & $5.48241 \cdot 10^{-8}$ \\
76015169 & -10443 & & 12078577080679 & -4122117 & $5.47191 \cdot 10^{-8}$ \\
184699341 & -17847 & & 18790887277234 & -4752656 & \\
281876941 & -19647 & & 20999693845505 & -5400411 & $3.73147 \cdot 10^{-8}$ \\
456877629 & -28531 & $1.42684 \cdot 10^{-6}$ & 29254665607331 & -6870529 & $2.06873 \cdot 10^{-8}$ \\
712638284 & -29736 & & 48136689451475 & -7816269 & \\
1122289008 & -43080 & & 72204113780255 & -11805117 & $-2.07576 \cdot 10^{-9}$ \\
1806141032 & -50356 & & 117374745179544 & -14496306 & \\
2719280841 & -62567 & & 176064978093269 & -17555181 & \\
\hline & & & & & \\
\hline
\end{tabular}

and here $T\left(n_{0}\right) \approx-1.0613886773 \cdot 10^{-14}$. It then immediately crosses back to a positive value, crosses back to negative again at $n_{0}+8$, reverts to positive at $n_{0}+56$, and oscillates more than 150000 times before beginning a lengthier succession of negative values beginning at $n_{0}+10532340211$. The minimum value of $T(n)$ over $n \leq 7.5 \cdot 10^{13}$ then occurs at $n_{1}=72204113780255$, as reported in Theorem 1 and

$$
T\left(n_{1}\right)=-0.00000000207576410927140618499378389075 \ldots
$$

The sum next achieves a positive value at $n=72213638492881$, and oscillates many more times up to $n_{2}=72234579516031$, its last crossing over this interval. In all, there are 327144 sign changes of the Turán sum below $7.5 \cdot 10^{13}$, all occurring between $n_{0}$ and $n_{2}$. Some successive local minima for $T(n)$, as well as $L(n)$, appear in Table 1.

Further analysis of the function $B_{m}^{*}(x)$ from (7) indicates that the next sign change for $T(n)$ (for $n>n_{2}$ ) may occur near $n=e^{43.897} \approx 1.159 \cdot 10^{19}$; see Figure 4. 


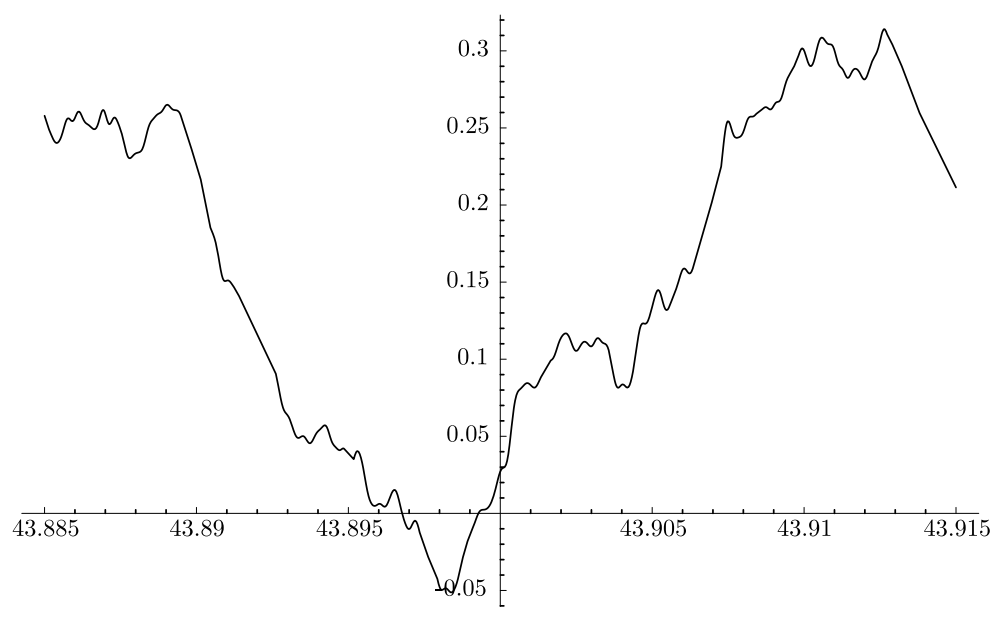

Figure $4 . B_{25000}^{*}(x)$ near $x=43.9$

\section{Sign changes in Pólya's sum}

It is an elementary fact that the summatory function of the Liouville function is nonzero precisely on the squares:

$$
\sum_{k \mid n} \lambda(k)= \begin{cases}1 & \text { if } n \text { is a square } \\ 0 & \text { otherwise }\end{cases}
$$

Lehman 12 exploits this fact to develop a method for computing $L(n)$ for a particular integer $n$ without computing $\lambda(k)$ for each $k \leq n$. We describe Lehman's formula here, and describe our strategy in implementing it to compute $L(n)$ for large $n$.

3.1. Lehman's method. From (13) we see that for any $x>0$ we have

$$
\lfloor\sqrt{x}\rfloor=\sum_{n \leq x} \sum_{k \mid n} \lambda(k)=\sum_{k \leq x} \lambda(k) \sum_{n \leq \frac{x}{k}} 1=\sum_{n \leq x} \sum_{k \leq \frac{x}{n}} \lambda(k)=\sum_{n \leq x} L\left(\frac{x}{n}\right) .
$$

Let $w$ be a positive real number satisfying $w<x$, and suppose $m$ is an integer between 1 and $\lfloor x / w\rfloor$. Replacing $x$ by $x / m$ in (14), then multiplying by the Möbius function $\mu(m)$ and summing over $m$, we obtain

$$
\sum_{m \leq \frac{x}{w}} \mu(m)\left\lfloor\frac{x}{m}\right\rfloor=\sum_{m \leq \frac{x}{w}} \mu(m) \sum_{n \leq x} L\left(\frac{x}{m n}\right) .
$$

We now break the right side into three sums for different ranges of $n$. We find that

$$
\begin{aligned}
& \sum_{m \leq \frac{x}{w}} \mu(m) \sum_{n \leq \frac{x}{m w}} L\left(\frac{x}{m n}\right)=\sum_{\ell \leq \frac{x}{w}} L\left(\frac{x}{\ell}\right) \sum_{m \mid \ell} \mu(m)=L(x), \\
& \sum_{m \leq \frac{x}{w}} \mu(m) \sum_{\frac{x}{m w}<n \leq \frac{x}{m v}} L\left(\frac{x}{m n}\right)=\sum_{\frac{x}{w}<\ell \leq \frac{x}{v}} L\left(\frac{x}{\ell}\right) \sum_{\substack{m \mid \ell \\
m \leq \frac{x}{w}}} \mu(m),
\end{aligned}
$$


and

$$
\sum_{m \leq \frac{x}{w}} \mu(m) \sum_{\frac{x}{m v}<n \leq \frac{x}{m}} L\left(\frac{x}{m n}\right)=\sum_{m \leq \frac{x}{w}} \mu(m) \sum_{k<v} \lambda(k)\left(\left\lfloor\frac{x}{k m}\right\rfloor-\left\lfloor\frac{x}{m v}\right\rfloor\right) .
$$

Combining these expressions, we see that

$$
\begin{gathered}
L(x)=\sum_{m \leq \frac{x}{w}} \mu(m)\left(\left\lfloor\sqrt{\frac{x}{m}}\right\rfloor-\sum_{k<v} \lambda(k)\left(\left\lfloor\frac{x}{k m}\right\rfloor-\left\lfloor\frac{x}{m v}\right\rfloor\right)\right) \\
\quad-\sum_{\frac{x}{w}<\ell \leq \frac{x}{v}} L\left(\frac{x}{\ell}\right) \sum_{\substack{m \mid \ell \\
m \leq \frac{x}{w}}} \mu(m) .
\end{gathered}
$$

In the same way, since

$$
\sum_{\substack{n \leq x \\ 2 \nmid n}} L\left(\frac{x}{n}\right)=\lfloor\sqrt{x}\rfloor-\left\lfloor\sqrt{\frac{x}{2}}\right\rfloor,
$$

one may obtain a somewhat more complicated formula for $L(x)$ that requires summing over only odd values of $\ell$ and $m$ :

$$
\begin{aligned}
L(x)=\sum_{\substack{m \leq \frac{x}{w} \\
2 \nmid m}} \mu(m)\left(\left\lfloor\sqrt{\frac{x}{m}}\right\rfloor\right. & -\left\lfloor\sqrt{\frac{x}{2 m}}\right\rfloor+\left\lfloor\frac{x}{2 m v}+\frac{1}{2}\right\rfloor \sum_{k<v} \lambda(k) \\
& \left.-\sum_{k<v}\left\lfloor\frac{x}{2 k m}+\frac{1}{2}\right\rfloor \lambda(k)\right)-\sum_{\substack{\frac{x}{w}<\ell \leq \frac{x}{v} \\
2 \nmid \ell}} L\left(\frac{x}{\ell}\right) \sum_{\substack{m \mid \ell \\
m \leq \frac{x}{w}}} \mu(m) .
\end{aligned}
$$

This is Lehman's formula for computing $L(x)$. One could in fact optimize the formula further, for instance by summing over only those integers $\ell$ and $m$ that are relatively prime to 6 , but this formula suffices for our purposes.

We now select $v$ and $w$ to minimize the resources required to calculate $L(x)$. The first sum requires values of the Möbius function up to $x / w$ and values of $L(n)$ for $n<v$, and we store these values in two arrays. To balance the space requirements here we then require $v w=x$. Using this data, the first sum then needs $O(v x / w)=O\left(v^{2}\right)$ time.

The second sum requires more care. Evidently we require values of $L(n)$ for $n<w$, but it is not necessary to store $O(w)$ values here. Instead, we need only store the values of $L(x / \ell)$ for odd integers $\ell$ in $(x / w, x / v]$. We therefore store the values of $L(n)$ for $n \leq \sqrt{x}$ in an array, and we insert the values of $L(x / \ell)$ for odd integers $\ell$ between $v$ and $\sqrt{x}$ in a search tree that guarantees $O(\log x)$ time for insertions and searches. Thus, the space requirement here is $O(\sqrt{x})$.

For the last part of the second sum, define the function $\xi(\ell)$ by

$$
\xi(\ell):=\sum_{\substack{m \mid \ell \\ m \leq \frac{x}{w}}} \mu(m) .
$$

We require values of $\xi(\ell)$ for odd integers $\ell \leq x / v$. We use a sieving strategy to compute the values of this function in blocks of size $q$, where $q$ is a free parameter. First, we initialize each element of an array of integers of size $q$ to 0 , then we add $\mu(3)=-1$ to each multiple of 3 in the range represented by the array, then 
$\mu(5)=-1$ to each multiple of 5 , and continue this for each odd integer $m \leq x / w$. By setting $q=\sqrt{x}$, we match the space requirement for the $L(n)$. Amortizing over the length of the interval, we see that this computation requires $O(\log x)$ time per value of $\xi(\ell)$.

With this strategy the time then required to compute the second sum is

$$
O\left(w \log x+\frac{x \log x}{v}\right)=O(w \log x),
$$

and the space needed is $O(\sqrt{x})$. We remark that we could reduce the space further by employing a sieving strategy in the computation of the $L(n)$, but for the applications of interest here, the table of values of $\lambda(n)$ for $n \leq 4 \cdot 10^{10}$ that was computed with Algorithm 1 suffices.

To minimize the time required, we thus set $v$ and $w$ so that $v w=x$ and $O\left(v^{2}\right)=$ $O(w \log x)$, so

and

$$
v=c(x \log x)^{1 / 3}
$$

$$
w=\frac{x^{2 / 3}}{c(\log x)^{1 / 3}},
$$

for a positive constant $c$, so that both sums require $O\left((x \log x)^{2 / 3}\right)$ time. We find empirically that choosing $c=1 / 2$ produces favorable computation times.

We remark that Deléglise and Rivat [3] developed a similar algorithm for computing the Mertens function $M(n)=\sum_{k=1}^{n} \mu(k)$.

3.2. Results. We used this algorithm to compute several values of $L(n)$. First, we used it to check particular values of $L(n)$ computed by Algorithm 2 on each interval of the computation. Second, we used this method, in combination with Algorithm 2, to find another range where $L(n)$ is often positive, and indeed quite large.

Prior to this work, the only known positive values of $L(n)$ occurred for $n<10^{9}$, in fact between $n_{1}=906150257$ and $n_{2}=906488079$. There are 133 sign changes of $L(n)$ for $n<10^{9}$ and, as Tanaka [17] observed, 252 integers in this range where $L(n)=0$, including the nine values of $n \leq 586$ found by Pólya where this occurs.

Some investigation of the approximating formula $A_{m}^{*}(x)$ from (4) indicates that the next crossing point for $L(n)$ probably occurs near $n=e^{33.492} \approx 3.511 \cdot 10^{14}$; see Figure 5. We use Lehman's formula and Algorithm 2 to verify that $L(n)$ indeed achieves positive values near this point. We first sample the values of $L(n)$ in this vicinity, then use Algorithm 2 to compute the values of $L(n)$ (but not $T(n)$ ) for $3.5 \cdot 10^{14} \leq n \leq 3.54 \cdot 10^{14}$. We find that $L(n)$ exhibits 159016 sign changes in this range; of these, 24715 occur between $n_{3}=351100332278253$ and $n_{4}=$ 351111026085887 , and the remaining 134301 sign changes occur between $n_{6}=$ 352716987837481 and $n_{7}=352748014189959$. A local maximum is achieved at

$$
n_{5}=351753358289465 \text {, }
$$

where

$$
L\left(n_{5}\right)=1160327 \text {. }
$$

Theorem 2 follows immediately from this value by using the results of Anderson and Stark [1], since $L\left(n_{5}\right) / \sqrt{n_{5}}=0.0618673 \ldots$.

This calculation detects 317060 integers in the range $3.5 \cdot 10^{14} \leq n \leq 3.54 \cdot 10^{14}$ where $L(n)=0$, beginning with $n_{3}-3=351100332278250$. Of these, 49590 


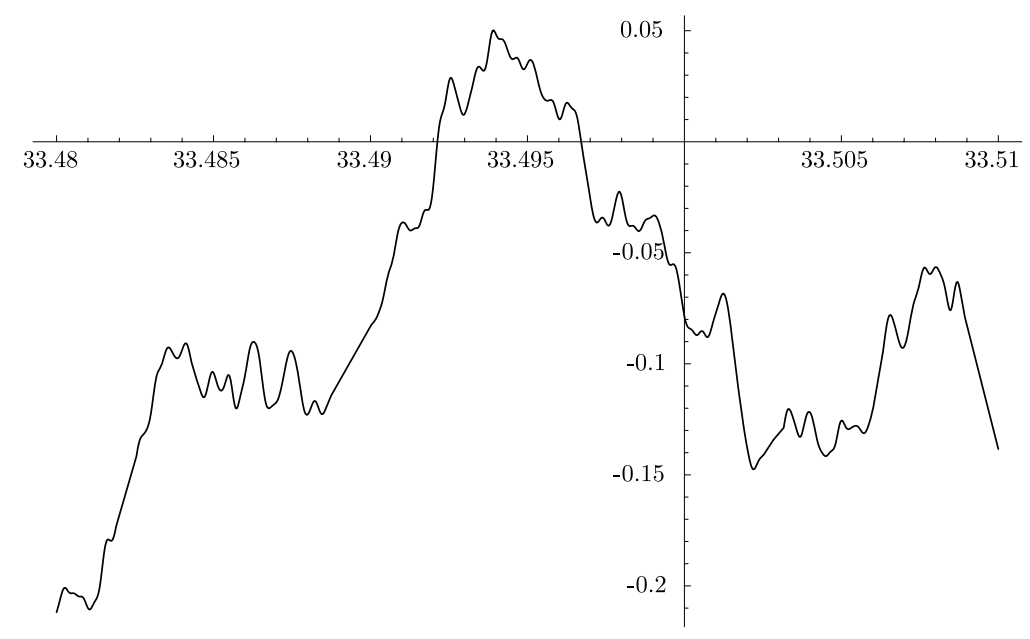

Figure 5. $A_{25000}^{*}(x)$ near $x=33.5$

occur between $n_{3}-3$ and $n_{4}+5$, and the other 267470 occur between $n_{6}-13$ and $n_{7}-1$. The complete list of 317312 known positive integers $n$ where $L(n)=0$ is available from the authors.

We remark that it is possible that $L(n) \geq 0$ for additional integers $n<3.5 \cdot 10^{14}$, although this seems unlikely, considering the values of the function $A_{m}^{*}(x)$. To resolve this in part, however, we used Algorithm 2 to extend the calculation of $L(n)$ (but not $T(n)$ ) up to $n=2 \cdot 10^{14}$. This computation required 1318 additional jobs, performed over 25 nights, using $n=8000$ blocks per process at first, reducing later to $n=6000$ and then $n=5000$. The total CPU time for this calculation was approximately 2.25 years. With this computation, we verified that indeed $L(n)<0$ for $10^{9} \leq n \leq 2 \cdot 10^{14}$.

\section{ACKNOWLEDGMENTS}

We thank several people for helping us to track down the history of prior computations on sums involving the Liouville function, especially Paul Bateman (Illinois), Ron Boisvert (NIST), and John Brillhart (Arizona). We also thank IRMACS and Simon Fraser University for computational resources, and we thank the referee for his or her careful reading of the manuscript.

\section{REFERENCES}

[1] R. J. Anderson and H. M. Stark, Oscillation theorems, Analytic Number Theory (Philadelphia, 1980), Lecture Notes in Math., vol. 899, Springer, Berlin, 1981, pp. 79-106. MR654520(83h:10082)

[2] P. T. Bateman and S. Chowla, The equivalence of two conjectures in the theory of numbers, J. Indian Math. Soc. (N.S.) 17 (1953), 177-181 (1954). MR0062176 (15:939c)

[3] M. Deléglise and J. Rivat, Computing the summation of the Möbius function, Experiment. Math. 5 (1996), no. 4, 291-295. MR1437219 (98f:11135)

[4] GMP: The GNU multiple precision arithmetic library. www.swox.com/gmp.

[5] A. Granville and K. Soundararajan, Negative values of truncations to $L(1, \chi)$, Proceedings of the Gauss-Dirichlet Conference (Göttingen, 2005), Clay Math. Proceedings, vol. 7, Amer. Math. Soc., Providence, 2007. 
[6] H. Gupta, On a table of values of $L(n)$, Proc. Indian Acad. Sci., Sect. A. 12 (1940), 407-409. MR.0003644 (2:248i)

[7] C. B. Haselgrove. Royal Society Depository for Unpublished Mathematical Tables, no. 65 (c. 1958).

[8] _ A disproof of a conjecture of Pólya, Mathematika 5 (1958), 141-145. MR 0104638 $(21: 3391)$

[9] H. D. Huskey, R. Thorensen, B. F. Ambrosio, and E. C. Yowell, The SWAC-design features and operating experience, Proc. Inst. Radio Engineers 41 (1953), 1294-1299. MR0061906 (15:902i)

[10] A. E. Ingham, On two conjectures in the theory of numbers, Amer. J. Math. 64 (1942), 313319. MR0006202 (3:271c)

[11] O. Larsen, Epreuve numérique d'une supposition de P. Turán, Mat. Tidsskr. B. 1952 (1952), 28. MR0053962(14:847c)

[12] R. S. Lehman, On Liouville's function, Math. Comp. 14 (1960), 311-320. MR0120198 $(22: 10955)$

[13] H. L. Montgomery, Zeros of approximations to the zeta function, Studies in Pure Mathematics: to the Memory of Paul Turán, Birkhäuser, Basel, 1983, pp. 497-506. MR820245 (87a:11081)

[14] M. R. Murty and A. Sankaranarayanan, Averages of exponential twists of the Liouville function, Forum Math. 14 (2002), no. 2, 273-291. MR.1880914 (2002m:11078)

[15] G. Pólya, Verschiedene Bemerkungen zur Zahlentheorie, Jahresber. Deutsch. Math.-Verein. 28 (1919), 31-40.

[16] R. M. Robinson, Mersenne and Fermat numbers, Proc. Amer. Math. Soc. 5 (1954), 842-846. MR.0064787 (16:335d)

[17] M. Tanaka, A numerical investigation on cumulative sum of the Liouville function, Tokyo J. Math. 3 (1980), no. 1, 187-189. MR584557 (83h:10010)

[18] P. Turán, On some approximative Dirichlet-polynomials in the theory of the zeta-function of Riemann, Danske Vid. Selsk. Mat.-Fys. Medd. 24 (1948), no. 17, 1-36. MR0027305 (10:286a)

[19] _ Nachtrag zu meiner Abhandlung "On some approximative Dirichlet polynomials in the theory of zeta-function of Riemann", Acta Math. Acad. Sci. Hungar. 10 (1959), 277-298. MR0115977 (22:6774)

[20] N. Wiener and A. Wintner, Notes on Pólya's and Turán's hypotheses concerning Liouville's factor, Rend. Circ. Mat. Palermo (2) 6 (1957), 240-248. MR0099319 (20:5759)

Department of Mathematics and Statistics, Simon Fraser University, Burnaby, B.C. V5A 1S6 CANADA

E-mail address: pborwein@cecm.sfu.ca

Department of Mathematics and Statistics, Simon Fraser University, Burnaby, B.C. V5A 1S6 CANADA

E-mail address: rferguson@pims.math.ca

Department of Mathematics, Davidson College, Davidson, North Carolina 28035-6996

E-mail address: mimossinghoff@davidson.edu 